\title{
Si può scrivere a registro?
}

\section{Massimo Bellina}

PUBBLICATO: 30 APRILE 2021

\section{Quesito:}

Una lettrice di Legnano ci segnala che nelle circolari scolastiche si leggono frequentemente le espressioni scrivere a registro e scrivere a diario, della cui correttezza dubita.

\section{Si può scrivere a registro?}

1 iportiamo anzitutto alcuni esempi dell'espressione:

Se proprio non fosse possibile avere elementi rispetto a questa competenza, si dovrà scrivere a registro che "Non è stato possibile procedere alla valutazione" (circolare del 27 marzo 2020, "Centro Provinciale per l'Istruzione degli Adulti", Verona);

Durante le giornate d'esame si consiglia ai docenti di scrivere a registro uscite e rientro del candidato (circolare del 7 giugno 20I2, Istituto Comprensivo "Morea-Tinelli", Alberobello);

I Docenti devono far scrivere a diario l'avviso e verificarne la presa visione con firma (circolare dell'8 ottobre 20I9, Istituto Comprensivo "Marcello Candia", Milano);

Ai Genitori delle classi $4 \wedge \mathrm{C} \mathrm{e} 4^{\wedge}$ B (dettare a diario) [...] Si prega di dare avviso scritto a diario con verifica di firma per presa visione da parte dei genitori (circolare del I8 maggio 20I6, Istituto Comprensivo "Margherita Hack", San Donato Milanese);

Agli studenti che manchino ai doveri scolastici inerenti alla disciplina sono inflitte le seguenti punizioni disciplinari [...]: richiamo scritto a registro di classe con convocazione della famiglia (I999, Regolamento disciplinare dell'Istituto di Istruzione Superiore "Giovanni Bertacchi", Lecco);

il rimprovero verbale e il rimprovero scritto (a diario o a registro elettronico) sono inflitti dal docente o dal Dirigente scolastico (20I8, Patto Educativo di corresponsabilità dell'ICS "Erasmo da Rotterdam", Cisliano, Milano).

Ciò che induce il dubbio sono due anomalie compresenti nelle espressioni in esame: la preposizione $a$ utilizzata per esprimere un complemento di stato in luogo (normalmente si utilizzano in o su: scrivere sul/nel registro) e l'assenza dell'articolo (richiesto anche in espressioni analoghe, del tipo pubblicare all'albo, sul sito ecc.).

Quelle che abbiamo appeno definito anomalie non sono tuttavia usi del tutto eccezionali nella nostra lingua: la preposizione $a$, normale con il complemento di termine, si usa comunemente anche con il complemento di stato in luogo nelle espressioni stare a scuola, rimanere a casa e simili, anche figurate e con verbi derivati proprio da scrivere (iscrivere alla classe prima, ascrivere a debito). Anche l'assenza dell'articolo si presenta in usi analoghi del tipo pubblicare in bacheca. In generale, osserviamo come nell'italiano contemporaneo esista una tendenza, forse di origine settentrionale, all'eliminazione degli 
articoli (settimana prossima, in Rai, ecc.).

Il giudizio sulla correttezza di queste forme è comunque delicato. Bisogna quindi valutare singolarmente ciascun caso, non solo prendendo in esame il criterio della coerenza logica e grammaticale sopra introdotto (e che in questo caso sembra in effetti debole), ma valutando anche i criteri dell'analogia con forme simili, della tradizione storica, dell'uso effettivo dei parlanti, della funzionalità dell'espressione nel sistema della lingua.

Con quest'ultimo punto intendo banalmente che occorre interrogarsi su questi aspetti: questo uso esprime il concetto meglio di quanto non facciano le forme concorrenti? La comunicazione fra i parlanti risulta più efficace o no? Suscita chiarezza o perplessità nei parlanti? Anche sotto questo riguardo sembra di poter rispondere negativamente.

Il criterio dell'analogia conduce a considerare l'esistenza di espressioni apparentemente affini e di uso corrente: oltre a quelle sopra rammentate, tenere a mente, mettere a bilancio, e altre con verbi derivati proprio da scrivere (ascrivere a debito/a merito/a biasimo: si noti tuttavia che in ascrivere la reggenza preposizionale è richiesta per coerenza con il prefisso $a$ - del verbo). Consideriamo ora il caso dell'espressione corrente iscrivere/iscritto al registro degli indagati, per rilevare anzitutto che di gran lunga maggiori sono le occorrenze di nel registro degli indagati, e in secondo luogo che ogni qual volta il complemento venga in qualche modo individuato da una specificazione (registro degli indagati, registro rosso, ecc.), ricorre motivatamente l'articolo determinativo. In italiano diciamo infatti, preferibilmente: competere in salita (perché l'ambito è generico e astratto), ma competere nello sci di fondo; essere bravo in matematica, ma essere bravo nello svolgimento degli esercizi.

Veniamo ora a considerare la diffusione nell'uso, che è argomento sempre decisivo: nei confronti della condivisione di una forma a poco servono, oggi meno che mai, le querele dei censori (si ricordi il celebre caso di piuttosto che). Ma l'espressione scrivere a registro (o a diario) non sembra affatto diffusa: è assente nei dizionari dell'uso e personalmente non ricordo di averla mai letta o sentita; non risulta nota neppure ad alcuni insegnanti di Roma da me interpellati. Le pochissime attestazioni presenti che ci procura la cornucopia di Google provengono pressoché esclusivamente da istituti scolastici della Lombardia o del Veneto (decine di attestazioni provengono invece dalle delibere amministrative del comune di Carmagnola, presso Torino, che evidentemente ripete lo stesso fac-simile per i propri atti). Alla nostra lettrice di Legnano non sarà sfuggito che provengono da scuole di Milano o dei suoi dintorni quasi tutti gli esempi citati e, in generale, pressoché tutti quelli rinvenuti in questa breve ricerca: Gallarate, Turbigo, San Donato, San Giuliano, Lecco, Cisliano, Melegnano, Codogno, Seregno. Di pochissimi esempi di altro genere darò notizia più avanti.

Se dall'uso presente passiamo alla tradizione storico-letteraria, la situazione non cambia: l'espressione è assente in tutte le edizioni del Vocabolario della Crusca; una sola attestazione figura nel TommaseoBellini (messa a registro; occasionalmente rileviamo in questo dizionario le forme mettere a libro, mettere ad entrata, notare a debito), due soltanto nel GDLI (far riconoscere e registrare le terre di questo monastero, che non sono ancora messe a registro).

L'indagine lessicografica ci presenta comunque qualche indizio interessante. L'espressione è infatti più volte documentata in dizionari dell'uso quasi tutti pubblicati in area lombarda fra fine Settecento e inizio Ottocento (anche se gli esempi sembrano per lo più ereditati l'uno dall'altro: pressoché in tutti ricorre identica l'espressione mettere, notare, scrivere a registro): Sinonimi ed aggiunti italiani (I756) di Carlo Costanzo Rabbi; Dizionario Universale Critico Enciclopedico (I797-1805) di Francesco Alberti di Villanuova; Nuovo dizionario italiano tedesco e tedesco italiano (I782) di Philipp-Jakob Flathe e Nicolo di 
Castelli (qui notiamo anche la variante scrivere a libro); Vocabolario piemontese-italiano (I832) di Michele Ponza.

Al termine di questa breve indagine, potremmo concludere che l'espressione sembra del tutto inusitata e comunque confinata in ambiti molto ristretti della lingua scritta dell'amministrazione, laddove ricorre spesso la predilezione consapevole per forme speciali o tecnicismi collaterali che gratificano soprattutto i burocrati. Tuttavia i pochi esempi che emergono dalla lessicografia e i rarissimi esempi contemporanei, in testi stilisticamente anche sorvegliati, ci consentono di distinguere, forse troppo sottilmente, due usi diversi dell'espressione.

Nei vocabolari sette-ottocenteschi scrivere a registro è sempre usato come sintagma verbale, ossia come espressione che i linguisti chiamano polirematica, fissata stabilmente in una determinata forma:

- riguardo al significato, significa 'riportare in un libro', ed è sinonimo di registrare, annotare, imputare, mettere in conto, con particolare riferimento a denaro, beni, quantitativi e simili, soprattutto in contesti amministrativi e contabili;

- riguardo alla forma, come tutte le locuzioni "cristallizzate", non ammette variazioni nell'ordine dei componenti né inserzioni o aggiunte: in particolare, una eventuale specificazione deve riguardare l'intera locuzione, non il singolo componente (posto che giacca a vento è una polirematica, non posso dire una giacca grande a vento, né una giacca a vento di tramontana).

Premesso questo, risultano a mio parere accettabili tutti i seguenti esempi contemporanei (a dire il vero, quasi gli unici che trovo):

la Juve si è resa disponibile a far scrivere a registro plusvalenze;

I motoveicoli usati invece hanno scritto a registro un bel io punti percentuali di positivo nel mese di Ottobre;

Ci sono molti modi di «distrarsi» dall'Immortalità, e molti codici per scrivere a registro la dolorosa perdita;

[Il ministro] ha evitato alle Arti un grave insulto / con un altro getton scritto a registro, / a favor di un anzian disoccupato (satira in endecasillabi di Carlo Cornaglia, torinese, scrittore e giornalista del "Fatto quotidiano").

In tutti questi esempi scrivere a registro è correttamente usato come sinonimo di registrare, in senso letterale o figurato; si osservi anche negli ultimi due anche il registro letterario. Si noti bene: registro risulta sempre imprecisato, ed è pertanto più giustificata l'assenza dell'articolo; è possibile sostituire con registrare senza alcun pregiudizio alla forma e al significato della frase.

Analizziamo ora invece gli esempi scolastici e amministrativi sopra richiamati:

richiamo scritto a registro di classe; richiamo scritto a registro di classe con convocazione della famiglia

Questi esempi suscitano immediatamente perplessità, senza bisogno di consapevolezza linguistica. Anzitutto qui non posso sostituire scritto a registro con registrato (l'esito della sostituzione sarebbe l'insensato richiamo registrato di classe). In secondo luogo, la specificazione di registro, in virtù della quale si determina un particolare tipo di registro, viola la consuetudine di utilizzo delle polirematiche: sottrae registro al sintagma verbale di cui è parte integrante, restituendolo come complemento di 
luogo a pieno diritto, espresso però stavolta in violazione della norma grammaticale per l'immotivata assenza dell'articolo e l'utilizzo anomalo della preposizione $a$.

si consiglia ai docenti di scrivere a registro uscite e rientro del candidato; Si invitano i coordinatori di classe a far effettuare il pre-test allegato prima degli incontri e di scrivere a registro l'impegno per [sic] le classi. (sic: meglio, gli impegni delle classi; circolare del 2 aprile 2org, Liceo Scientifico "G. B. Benedetti", Venezia)

Questi due casi sono diversi dal precedente: la sostituzione di registrare a scrivere a registro produce forme grammaticalmente corrette, ma imperfette sul piano della comunicazione: verrebbe infatti perduta l'informazione significativa che con registro si intende, ellitticamente e in ragione del contesto, il registro di classe.

scrittura privata [...] scritta a registro negli atti non soggetti a registrazione di questa Amministrazione appaltante. (formula ricorrente in molte delibere del Comune di Carmagnola)

Anche in questo caso la sostituzione è possibile (registrata negli atti ecc.); tuttavia l'espressione scritta a registro negli atti è ambigua per la distinzione fittizia di due complementi di stato in luogo, e dovrebbe essere cosi regolarizzata: scritta nel registro degli atti (con complemento unico e rispetto della norma).

E analogamente: "Con iscrizione a Registro fondiario del I2 dicembre $20 \mathrm{I} 2$ il fondo è stato venduto" (2015, Comune di Minusio, presso Locarno).

In conclusione, diremmo che questi ultimi casi siano da considerare non consigliabili, accertata la mancanza di motivazione sui diversi piani dell'uso, logico-grammaticale, storico e funzionale.

Più arduo ancora è trovare argomenti a favore della variante scrivere a diario, che non sembra disporre dei margini di accettabilità che abbiamo in alcuni casi riscontrato in scrivere a registro: non possiede un corrispondente sinonimico (come invece per mettere a registro $=$ registrare) e non ha attestazioni nel patrimonio lessicografico; anche le occorrenze tratte da Internet sono francamente esigue e poco significative: unicamente circolari emesse in istituti milanesi. Da notare tuttavia alcuni esempi di un uso diverso di scrivere/scritto a diario, riferito a libro che sia scritto "in forma di diario": "scritto a diario, si legge tutto di un fiato e con il cuore gonfio di Bellezza"; "la trama del libro - scritto a diario, con SMS, Facebook e WhatsApp - parla non solo dell'amore" ecc.; un'attestazione nobile è nello scrittore torinese Giampaolo Barosso: "Visto che a scrivere il diario invece non ho problemi, potrei scrivere a diario anche l'autobiografia" ( $A A A$, vol. III, 200I, prima ediz. 1996).

È possibile che l'espressione scrivere a diario, come in uso in alcune scuole lombarde, sia ricalcata su scrivere a registro, in virtù di un processo di estensione analogica favorito dal comune contesto d'uso dei referenti (comunicazioni e circolari vengono spesso registrate insieme sul diario degli alunni e sul registro di classe, quest'ultimo a volte anche denominato diario o giornale di classe).

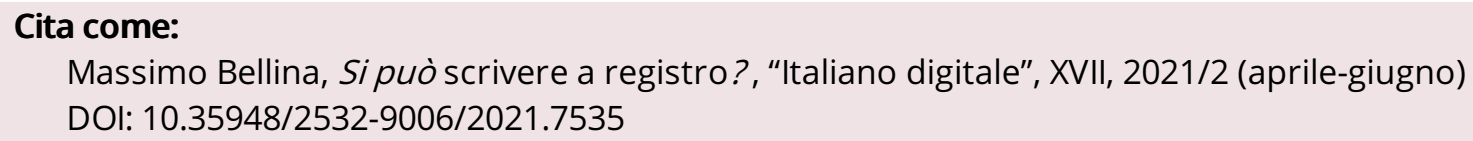

